\title{
Effect of Hydrostatic Pressure on the Refractive Indices of Some Solids
}

\author{
R. M. Waxler and C. E. Weir
}

(March 3, 1965)

\begin{abstract}
Measurements were made on refractive index changes with hydrostatic pressures between 1 bar and $1 \mathrm{kbar}$ using the helium yellow line. The materials studies were: $\mathrm{KBr}$ $\mathrm{NaCl}, \mathrm{LiF}$, diamond, $\mathrm{MgO}$, quartz, $\mathrm{Al}_{2} \mathrm{O}_{3}$, and three silicate glasses. All the materials increased in refractive index with pressure except diamond, $\mathrm{MgO}$, and $\mathrm{Al}_{2} \mathrm{O}_{3}$ which decreased, and LiF which did not change. The results were compared with photoelastic measurements, and Pockel's geometric theory of photoelasticity was substantiated as well as Mueller's physical theory. The data show that the ratio of change of polarizability with density is greater for solids having stronger interatomic repulsive forces. Volume and temperature coefficients of polarizability were evaluated for the cubic crystals and glasses. The thermooptic behavior of crystals and glasses is discussed.
\end{abstract}

\section{Introduction}

The relationship between index of refraction and density is of importance because of the effect of atomic interactions on the atomic polarizabilities. For transparent solids, values of $\rho d \mu / d \rho$, where $\mu$ is the index and $\rho$ the density, have been obtained by calculation from the photoelastic constants [1]. ${ }^{1}$ For crystals as well as glasses values of $\rho d \mu / d \rho$ so calculated usually have been found to be less than the corresponding values calculated on theoretical grounds, i.e., from the Lorentz-Lorenz and the Drude relationships [1]. The most extreme example of this discrepancy is probably found in the case of diamond and $\mathrm{MgO}$ where $\rho d \mu / d \rho$ values calculated from photoelastic constants are negative, a result which is clearly impossible to reconcile with the assumption of constant polarizability involved in the initial derivation. The theory of photoelasticity due to Mueller $[2,3]$ recognizes the change in the intrinsic polarizability of an atom due to strain.

For glasses, data on $\rho d \mu / d \rho$ have been obtained by Ritland [4] (and others) in the annealing temperature range where both index and density change with time at constant temperature and on volumetric relaxation following removal of high hydrostatic pressures [5]. In the former case $\rho d \mu / d \rho$ is found to be somewhat less than theory predicts and in the latter case somewhat greater.

Direct experimental data on $\rho d \mu / d \rho$ are required to understand thermo-optic properties. Values of $d \mu / d T$, where $T$ is the temperature, have been measured for many solids and it is known that the sign may be either positive or negative. For most crystals $d \mu / d T$ is found to be negative but in the case of diamond, $\mathrm{MgO}$, and $\mathrm{ZnS}$ the reverse is true [6]. Recent work has shown that for $\mathrm{Al}_{2} \mathrm{O}_{3} \quad d \mu / d T$ is positive for both the ordinary and extraordinary

\footnotetext{
1 Figures in brackets indicate the literature references at the end of this paper.
}

rays [7]. Most optical glasses exhibit a minimum in the $\mu$ - $T$ curve somewhat below room temperature so that at and above room temperature $d \mu / d T$ is positive but at some lower temperature the sign reverses [8]. Any attempt to explain such a complex thermo-optic behavior must of necessity require an understanding of the $\rho d \mu / d \rho$ effect because thermal dilatation is an important contributing factor to the value of $d \mu / d T$.

Finally, by reversal of the customary procedure, data on $\rho d \mu / d \rho$ may be used to evaluate photoelastic constants in certain instances. For glasses only two constants are required, so that knowledge of $\rho d \mu / d \rho$, plus information on the optical path difference produced by a unidirectional stress serves to determine completely the photoelastic constants.

For these several reasons it was considered of interest to obtain direct measurements of the density coefficient of refractive index, $\rho d \mu / d \rho$, in solids at constant temperature by the application of hydrostatic pressure. This is the first time that such an approach has been used. This report contains data on $\rho d \mu / d \rho$ obtained in the pressure interval between 1 bar and approximately 1000 bars for several crystals and glasses of interest. Data were obtained from specimens of the cubic ionic crystals, $\mathrm{LiF}, \mathrm{KBr}$, $\mathrm{NaCl}$, and $\mathrm{MgO}$; the covalent cubic crystal, diamond; and the birefracting crystals, $\mathrm{Al}_{2} \mathrm{O}_{3}$ and quartz where both the ordinary and extraordinary rays were studied. The glasses studied were fused silica, a commercial plate glass and a borosilicate crown glass, BSC 517/645. Measurements reported here were made only at $25^{\circ} \mathrm{C}$ using the helium yellow line.

\section{Experimental Method}

In these experiments interference fringes were observed in a plate of the material having plane, polished, nearly parallel faces [9]. The fringes were viewed in reflection using collimated helium light of 
$\lambda=5875.62 \AA$ at normal incidence. For a specimen of thickness, $t$, and index, $\mu$, the fringe number $N$ is given by the expression

$$
N \lambda=2 t \mu .
$$

Application of hydrostatic pressure will produce a change in $\mu$, a decrease in $t$, and a shift in the interference fringes. By differentiating (1) and rearranging it is found that

$$
\Delta \mu=\frac{\Delta N \lambda}{2 t}-\mu \frac{\Delta t}{t}
$$

A measurement of the number of fringes that pass a point of reference, and the thickness together with data on the initial index and the linear compression $\Delta t / t$ permits calculation of the change in index.

The experimental apparatus and method have been described earlier in detail and will only be outlined here [10]. Briefly, a plate of the test specimen is immersed in liquid in a pressure vessel equipped with glass windows. Hydrostatic pressure is generated by compressing the liquid and the number of interference fringes passing a reference line, i.e., the fringe count, was determined visually. Estimates of the number of fringes were made to the nearest tenth of a fringe.

The change in fringe number observed in approximately 1 kbar is not very large for these solids because of their rather low compressibilities compared to the liquids previously studied and because the decrease in thickness under pressure acts in such a direction as to decrease the fringe number (see eq (2)). In the most favorable case $\Delta N$ is known only to three significant figures. Extreme precision in the other quantities is not required. The most reliable values for the initial indices were used, however, and values for the index at the frequency of the He yellow line were generally obtained by interpolation of the dispersion data reported. The data on the indices of refraction of the solids may be found in references [11] through [20]. For BSC $517 / 645$ the index was reported for the sodium D line only. However, from the low dispersion of such glasses and the small frequency separation of the sodium and helium yellow lines, the value reported was assumed to apply to the present measurements within the required precision.

Compression data for all materials except BSC $517 / 645$ have been reported, usually in terms of volume compression $\left(\Delta V / V_{0}\right)$. Volume compressions were converted to linear values for the isotropic materials through the relationship $\Delta t / t_{0}=\frac{\Delta V}{3 V_{0}}$. For quartz and $\mathrm{Al}_{2} \mathrm{O}_{3}$, where data on linear compressions are available, $\Delta t / t_{0}$ was computed for the direction perpendicular to the optic axis. The data for determining the compression of the solids may be found in references [21] through [26]. (For BSC 517/645 the linear compression was calculated from elastic constant data measured at 1 bar [27].) It has been estimated that, at most, the uncertainty in values of $\Delta t / t_{0}$ lies in the third significant figure.
For the optically isotropic solids (cubic crystals and glasses) the measurements were straightforward but for the crystals of lower symmetry, $\mathrm{Al}_{2} \mathrm{O}_{3}$ and quartz, which are optically anisotropic the method was modified to permit measurements for both the ordinary and extraordinary ray. For these materials the specimen was oriented so that the optic axis was perpendicular to the direction of the light beam. In the measurements a large polarizer was inserted between the source and the window of the pressure vessel. On rotating the polarizer two distinct fringe systems could be seen depending on whether the electric vector of the plane polarized light transmitted by the polarizer was parallel or perpendicular to the optic axis of the specimen. (For the quartz crystal which is optically active, it should be noted that, since the fringes were obtained by reflection, the emergent polarized light was still plane polarized in the same plane at which it was incident [28]. This would not have been true if the fringes were observed in transmission [16].) On applying hydrostatic pressure the fringe shift for each separate fringe system was noted, giving data on both rays for the birefringent crystals.

\section{Preparation of Specimens}

Most specimens were prepared by sectioning or clearing larger samples of commercially available synthetic single crystals. However, both quartz and diamond were prepared from naturally occurring single crystals. The glasses were of commercial origin. Although the test specimen required was approximately $1 \mathrm{~cm} \times 1 \mathrm{~cm} \times 0.5 \mathrm{~cm}$, it was found expedient to grind and polish larger specimens and then to cut the final specimen from the most perfectly ground portion of the large piece. Rough blanks of both $\mathrm{Al}_{2} \mathrm{O}_{3}$ and quartz were cut witb the optic axes in approximately the correct orientation. The blanks were then oriented using the Laue back-reflection x-ray technique and ground so that the optic axis was in the surface of the specimen.

\subsection{Grinding and Polishing}

A brief account is given here of the preparation of the crystals because these techniques are not widely known. The specimens were first ground flat and with nearly parallel surfaces using a surface grinder for the harder materials and using hand grinding with fine abrasive for the alkali halides. The harder crystals were prepolished on a wood lap with $8 \mu-22 \mu$ diamond powder using olive oil lubricant and then given a final polish on cloth impregnated with $3 \mu$ diamond dust using an alcohol-water solution as a lubricant. The alkali halides were given only the latter of the two polishing steps. $\mathrm{Al}_{2} \mathrm{O}_{3}$ specimens were available only in small diameters and the grinding and polishing operation was conducted on an assemblage of five small disks, set in wax on a flat glass plate.

The diamond was a 2 carat brilliant cut gemstone and the table face was found to be satisfactory for one surface. The culet was ground down parallel to 
the table face using a high speed steel lap impregnated with diamond powder to produce a surface approximately $1 \mathrm{~mm}$ in width. Satisfactory fringes could be observed through this small surface.

In all polishing operations it was necessary to check the fringe system at frequent intervals. If it was noted that the surfaces were deviating too much from parallelism adjustments were made in the polishing to reduce the angle between the surfaces.

\subsection{Aluminizing}

In order to improve the sharpness of the interference fringes the reflecting power of the surfaces of the specimens was increased by vacuum deposition of a layer of aluminum. The front surface of each specimen was covered with a partly reflecting film while the rear surface was made fully reflecting. Multiple reflection fringes were obtained in this manner but because of the large thickness to length ratio of the specimens the fringes were not extremely sharp. A series of depositions of different thicknesses were made on the front surface of plate glass specimens until the fringes appeared to reach a maximum intensity and sharpness. This film thickness was found to be satisfactory for all other specimens.

TABLE 1. Pressure induced changes in refractive index as measured with helium yellow radiation of $5875.62 \breve{A}$

\begin{tabular}{|c|c|c|c|c|c|c|}
\hline Material & $\begin{array}{c}\text { Refractive } \\
\text { index, } \\
\mu_{\mathrm{He}}\end{array}$ & $\begin{array}{c}\text { Specimen } \\
\text { thickness, } \\
t_{0}\end{array}$ & $\begin{array}{l}\text { Pressure, } \\
P\end{array}$ & $\begin{array}{l}\text { Thickness } \\
\text { change, } \\
-\Delta t / t_{0} \times 10^{3}\end{array}$ & $\begin{array}{c}\text { Observed } \\
\text { change in } \\
\text { fringe number } \\
-\Delta N\end{array}$ & $\begin{array}{l}\text { Change in } \\
\text { refractive } \\
\text { index } \\
\Delta \mu \times 10^{5}\end{array}$ \\
\hline $\mathrm{KBr}_{-}$ & 1. 560 & $\begin{array}{l}C m \\
0.3210\end{array}$ & $\begin{array}{l}\text { bars } \\
\quad 966.1\end{array}$ & & & \\
\hline $\mathrm{NaCl}$. & $\begin{array}{l}\text { 1. } 546 \\
\text { la }\end{array}$ & .6748 & 963. 6 & 1. 348 & $\begin{array}{l}11.3 \\
21.6\end{array}$ & $\begin{array}{l}226 \\
115\end{array}$ \\
\hline $\mathrm{LiF}_{\ldots} \ldots$ & 1.392 & .5683 & 963.2 & .349 & 9.4 & 0 \\
\hline $\mathrm{MgO}_{\ldots}$ & 1.738 & .3970 & 960.8 & .190 & 6.8 & -17 \\
\hline Diamond & 2. 418 & .4037 & 960.0 & .058 & 3.4 & -11 \\
\hline $\begin{array}{l}\text { Quartz: } \\
\quad \omega_{-}\end{array}$ & 1.545 & .5112 & 957.6 & .944 & 6.9 & \\
\hline $\begin{array}{r}\epsilon \\
\text { Sapphire: }\end{array}$ & 1. 554 & .5112 & 961.5 & .948 & 7.5 & 107 \\
\hline$\omega$ & 1. 769 & .5659 & 961.8 & .105 & 6.2 & -14 \\
\hline & 1. 760 & .5659 & 957.6 & .105 & 6.2 & -14 \\
\hline Fused $\mathrm{SiO}$ & 1.458 & . 4967 & 960.2 & .865 & 7.3 & 83 \\
\hline BSC $517 / 6$ & 1. 517 & . 6693 & 960.1 & .717 & 8.9 & 70 \\
\hline Plate glass_. & 1.518 & . 6566 & 960.0 & .723 & 9.1 & 69 \\
\hline
\end{tabular}

\section{Results}

The results of the experiments are given in table 1 which shows the decrease in fringe number, the dimensional change calculated at the pressure given and the change in index of refraction. It should be moted that the change in fringe number is presented in table 1 for the direction of increasing pressure, i.e., between 1 bar and the pressure listed. For birefracting crystals the index for the ordinary and extraordinary rays are noted by $\omega$ and $\epsilon$ respectively. Each value of $\Delta N$ given in table 1 represents the average of at least two determinations. Such duplicate measurements agreed to within \pm 0.1 interference fringe. From the agreement between the duplicate measurements the limit of reproducibility in the index change is estimated to be $\pm 2 \times 10^{-5}$.

From the tabular data it is seen that the change in index is quite small in absolute value, varying from 0 in $\mathrm{LiF}$ to 2 in the third decimal place for $\mathrm{KBr}$. The index decreases for $\mathrm{MgO}$, diamond and both the ordinary and extraordinary rays of $\mathrm{Al}_{2} \mathrm{O}_{3}$. All other materials show an increase in index except LiF for which the index is unchanged. For the birefracting crystals both the ordinary and extraordinary rays show the same change in index within the limits of error.

\section{Discussion}

\subsection{Cubic Crystals}

From the experimental values of $\triangle \mu$ and the compressibility data, it was possible to calculate values for the quantity $\rho \frac{\Delta \mu}{\Delta \rho}$ where $\rho$ is the density. For the cubic crystals studied here the theory of photoelasticity shows that $\rho \frac{d \mu}{d \rho}$ can be calculated by the relationship

$$
\rho \frac{d \mu}{d \rho}=\frac{\mu^{3}}{6}\left(p_{11}+2 p_{12}\right),
$$

where the elasto-optic constants, $p_{i j}$, relate the state of strain existing in a crystal to changes in the index ellipsoid [6]. These values have been calculated for a number of crystals $[1,6]$ and the results are given in table 2 together with the initial indices, $\mu$. [The values of these experiments obtained over the pressure interval of approximately $1 \mathrm{~kb}$ are denoted $\rho \Delta \mu / \Delta \rho$ while those from the photoelastic theory are designated as $\rho d \mu / d \rho]$. It may be noted that the photoelastic data were obtained for the sodium D line and the present data for the helium yellow line. However, because of the small frequency difference between the two lines and the known low-frequency dependence of $\rho d \mu / d \rho[29,30]$ any differences arising from dispersion effects can be taken to be negligible. 


$$
\text { TABLE 2. Values of } \rho\left(\frac{\Delta \mu}{\Delta \rho}\right) \text { and } \lambda_{\mathrm{o}} \text { for cubic crystals and }
$$

\begin{tabular}{|c|c|c|c|c|c|c|c|}
\hline \multirow{2}{*}{ Material } & \multirow{2}{*}{$\begin{array}{c}\text { Refrac- } \\
\text { tive } \\
\text { index, } \\
\mu_{0}\end{array}$} & \multirow{2}{*}{$\rho\left(\frac{\Delta \mu}{\Delta \rho}\right)$} & \multirow{2}{*}{$\rho\left(\frac{d \mu}{d \rho}\right)$} & \multicolumn{2}{|c|}{ L-L equation } & \multicolumn{2}{|c|}{ Drude equation } \\
\hline & & & & $\rho\left(\frac{\partial \mu}{\partial \rho}\right)$ & $\lambda_{o}$ & $\rho\left(\frac{\partial \mu}{\partial \rho}\right)$ & $\lambda_{o}$ \\
\hline $\begin{array}{l}\mathrm{KBr} \\
\mathrm{NaCl} \\
\mathrm{LiF} \\
\mathrm{MgO} \\
\text { Diamond } \\
\text { Fused } \mathrm{S}_{1} \mathrm{O}_{2} \\
\mathrm{BSC} 517 / 645^{-.} \\
\text {Plate glass }\end{array}$ & $\begin{array}{l}1.559 \\
1.544 \\
1.392 \\
1.736 \\
2.417 \\
1.458 \\
1.517 \\
1.518\end{array}$ & $\begin{array}{r}0.35 \\
.28 \\
.00 \\
-.31 \\
-1.58 \\
.32 \\
.33 \\
.32\end{array}$ & $\begin{array}{c}0.35 \\
.24 \\
.1 \\
-.40 \\
-.28 \\
.29\end{array}$ & $\begin{array}{r}0.68 \\
.66 \\
.44 \\
.97 \\
2.62 \\
.53 \\
.62 \\
.62\end{array}$ & $\begin{array}{r}0.48 \\
.58 \\
1.00 \\
1.32 \\
1.60 \\
.40 \\
.47 \\
.49\end{array}$ & $\begin{array}{r}0.46 \\
.45 \\
.34 \\
.52 \\
1.00 \\
.39 \\
.43 \\
.43\end{array}$ & $\begin{array}{r}0.24 \\
.38 \\
1.00 \\
1.60 \\
2.58 \\
.17 \\
.24 \\
.26\end{array}$ \\
\hline
\end{tabular}

A comparison of the figures in table 2 shows that there is essential agreement between the predictions of photoelastic theory developed by Pockels [31] and the actual measured values. Considering the errors inherent in the measurements of the photoelastic constants [6], i.e., small fringe shifts, nonuniformity of stress distribution in uniaxial compression, etc., it is considered that the agreement in table 2 is satisfactory except in the case of diamond. For this material, however, the errors in the photoelastic experiments and in the present work are likely to be particularly large because of the small specimens available and the small changes that are observed. There is no question as to the negative sign of $\rho \Delta \mu / \Delta \rho$ for diamond, and it is interesting to note that the results of the present experiments bear out the theoretical predictions of negative values of $\rho \Delta \mu / \Delta \rho$ for both $\mathrm{MgO}$ and diamond.

The data for diamond obtained in this study are in much better agreement with the results of Gibbs and Hill [32] on the change of the dielectric constant of diamond with pressure. Gibbs and Hill noted that the dielectric constant of diamond, $\epsilon=5.66$ at $27{ }^{\circ} \mathrm{C}$ at frequencies as high as $10 \mathrm{MHz}$ is equal to the square of the optical index of refraction at infinite wavelength, $\mu_{\infty}$, reported by Peter [14]. Since $\rho$ is proportional to $1 / V,(\partial \ln \rho / \partial P)_{T}=-(\partial \ln V / \partial P)_{T}=\beta$, where $P$ is the pressure and $\beta$ the compressibility. It follows that

$$
\rho\left(\frac{\partial \mu_{\infty}}{\partial \rho}\right)_{T} \equiv\left(\frac{\partial \mu_{\infty}}{\partial P} \frac{\partial P}{\partial \ln \rho}\right)_{T}=\frac{1}{\beta}\left(\frac{\partial \mu_{\infty}}{\partial P}\right)_{T}^{2}
$$

If $\epsilon=\mu_{\infty}^{2}$, it follows that

$$
\frac{1}{\mu_{\infty}}\left(\frac{\partial \mu_{\infty}}{\partial P}\right)_{T}=\frac{1}{2 \epsilon}\left(\frac{\partial \epsilon}{\partial P}\right)_{T} .
$$

If the value of $\rho\left(\frac{\Delta \mu}{\Delta \rho}\right)$ in the present study is used for $\rho\left(\partial \mu_{\infty} / \partial \rho\right)_{T}$ in eq (4), and employing the equivalence shown in eq $(5),(1 / \epsilon)(\partial \epsilon / \partial P)_{T}$ for diamond has been found to have the value $-2.28 \times 10^{-7}$ bar $^{-1}$. The value obtained experimentally by Gibbs and Hill for a type I diamond is $-2.40 \times 10^{-7} \mathrm{bar}^{-1}$.

\footnotetext{
2 The derivation leading to eq (4) was suggested by D. D. Wagman of NBS.
}

\subsection{Glasses}

Experimental values for $\rho \Delta \mu / \Delta \rho$ for the glasses are given in table 2 . The values are very similar for all three glasses and probably reflect the fact that the data are determined largely by the $\mathrm{SiO}_{4}$ tetrahedra common to all three glasses. Numerical comparisons with photoelastic theory can be made only for vitreous $\mathrm{SiO}_{2}$ for which Jog [29] has reported the necessary photoelastic data. As seen in the table the value of $\rho d \mu / d \rho$ calculated from the photoelastic constants is in reasonably good agreement with the value obtained here by direct measurement. All three glasses show positive values for $\rho \Delta \mu / \Delta \rho$. Furthermore the photoelastic data on numerous optical glasses reported by Schaeffer and Nassenstein [33], Vedam [34], and Mueller [3] invariably indicate positive values for $\rho d \mu / d \rho$. This fact will be discussed later.

\subsection{Noncubic Crystals}

It is interesting to note that the increase in index for both rays in quartz is greater than observed in fused silica. The result can be explained only by the conclusion that interatomic interactions play an effective role in the index change with density and that those are different in the crystal and the glass because of the crystal structure and the higher initial density of the crystal. From the data of table 1 it appears that the extraordinary ray suffers the larger change in index but the difference is probably within the experimental error. Pockels [35] has measured the photoelastic constants of quartz and his results can be extrapolated linearly to the present pressure of 957 bars to permit calculation of $\Delta \mu$. Under these circumstances the photoelastic data yield calculated values for the change of index of $100 \times 10^{-5}$ for the ordinary ray and $104 \times 10^{-5}$ for the extraordinary ray. These values are in good agreement with the results of the present studies. In contrast to quartz both rays of sapphire show the same change in index but both are negative. There do not appear to be enough photoelastic data available to permit calculations of the index change for $\mathrm{Al}_{2} \mathrm{O}_{3}$ [7].

\subsection{Photoelastic Constants of Glass}

From the present data on $\Delta \mu / \Delta P$ and auxiliary data on the amount of double refraction introduced into glass by uniaxial compression it is possible to compute the constants $q_{11}$ and $q_{12}$, where the piezooptic constants, $q_{i j}$, relate the state of stress which exists in a solid to changes in the index ellipsoid [6]. The relationships used [34] are as follows:

$$
\Delta \mu / \Delta P=\mu^{3}\left[q_{11}+2 q_{12}\right] / 2
$$

and

$$
\left(\Delta \mu_{2}-\Delta \mu_{1}\right) / \Delta P=\mu^{3}\left[q_{12}-q_{11}\right] / 2,
$$

where, in addition to the terms previously defined, $\Delta \mu_{2}$ and $\Delta \mu_{1}$ represent changes in index for light polarized respectively perpendicular and parallel to 
the stress direction. (In eq ( 7$), \Delta P$ denotes a change in a compression applied in one direction only.) The quantity $\left(\Delta \mu_{2}-\Delta \mu_{1}\right) / \Delta P$ is defined as the relative stress optical coefficient and has been reported to be 2.81 brewsters for BSC 517/645 [36] and 2.62 brewsters for plate glass [37]. These data plus values of $\Delta \mu / \Delta P$ serve to determine values for $q_{11}$ and $q_{12}$ from eq (6) and eq (7). These values are given in table 3. Values of Neumann's photoelastic constants, $p$ and $q$, are also given in table 3 ; the relationships used in evaluating them are given by Vedam [34].

The elasto-optic constants, $p_{11}$ and $p_{12}$ can also be calculated from the relationships given by Vedam [34]. Values of the elastic moduli needed in the calculations are given by Spinner for BSC 517/645 [27]. From measurements made on commercial plate glass [38], values for Young's modulus, the rigidity modulus and Poisson's ratio have been found to be $723.9 \mathrm{kbar}, 297.9 \mathrm{kbar}$, and 0.215 respectively. These values were used to calculate the elasto-optic constants for plate glass shown in table 3 .

TABLE 3. The photoelastic constants of BSC 517/645 and plate glass

\begin{tabular}{|c|c|c|c|c|c|c|}
\hline Glass & $p$ & $q$ & $p_{11}$ & $p_{12}$ & $q_{11} \times 10^{13}$ & $q_{12} \times 10^{13}$ \\
\hline BSC $517 / 645 \ldots$ & 0.168 & 0.087 & 0.115 & 0.221 & $\begin{array}{r}\mathrm{cm}^{2} / \text { dyne } \\
0.315\end{array}$ & $\begin{array}{r}\mathrm{cm}^{2} / \text { dyne } \\
1.92\end{array}$ \\
\hline Plate glass... & .154 & .087 & .114 & .203 & .369 & 1.87 \\
\hline
\end{tabular}

\subsection{Polarizability and Density}

It seems self-evident that density changes must be accompanied by some change in molecular or atomic polarizability and it is useful to analyze the present data to ascertain what information can be obtained in this connection. The most comprehensive theory appears to be due to Mueller [2,3] who considers the following factors involved in change of refractive index of a solid under applied stress: (1) change of density; (2) change of the Lorentz-Lorenz field; (3) change of the coulomb field; and (4) change in the intrinsic polarizabilities of the atoms. Mueller appears to be the first investigator to recognize the importance of the last factor. The theory has been applied so far only to cubic crystals and isotropic solids because in these cases the symmetry is such that the coulomb field can be taken to be zero. Under hydrostatic pressure the symmetry is unchanged and the coulomb field remains zero but the Lorentz-Lorenz field may change because of the change in density.

There are two principal theoretical relationships relating the index and density, the Lorentz-Lorenz,

and the Drude,

$$
\frac{\mu^{2}-1}{\mu^{2}+1}\left(\frac{1}{\rho}\right)=\alpha_{1}
$$

$$
\left(\mu^{2}-1\right) / \rho=\alpha_{2}
$$

where $\alpha_{1}$ and $\alpha_{2}$ are constants. In deriving the
Lorentz-Lorenz equation the local field has been accounted for through the theoretical value $4 / \pi 3 \bar{P}$ where $\bar{P}$ is the polarization of the medium and the change in local field with density is accounted for in the equation. The change in the local field with density has been ignored in the Drude equation. ${ }^{3}$

If $\alpha_{1}$ and $\alpha_{2}$ are rigorously constants eq (8) and eq (9) may be differentiated to obtain the change of index with density. This was apparently first done by Pockels [39] who obtained the two expressions

$$
\begin{aligned}
& \rho \frac{\partial \mu}{\partial \rho}=\left(\mu^{2}-1\right)\left(\mu^{2}+2\right) / 6 \mu \\
& \rho \frac{\partial \mu}{\partial \rho}=\left(\mu^{2}-1\right) / 2 \mu
\end{aligned}
$$

corresponding to the Lorentz-Lorenz and Drude formulations respectively. In eq (10) and eq (11) partial derivatives are used to indicate that there may be a change in atomic polarizability which is neglected. Mueller [2, 3] introduced the strain polarizability parameter, $\lambda_{0}$, to evaluate the change in polarizability by means of the relationship,

$$
\rho d \mu / d \rho=\left(1-\lambda_{0}\right) \rho \partial \mu / \partial \rho .
$$

This relationship follows from considering $\alpha_{1}$ and $\alpha_{2}$ to be dependent on the polarizability. By using eq (12) in conjunction with eqs (10) and (11) and the experimental values of $\rho \Delta \mu / \Delta \rho$ values of $\lambda_{0}$ have been calculated for both the Lorentz-Lorenz and the Drude formulations. These data are shown in table 2.

Burstein and Smith [1] have proposed that $\lambda_{0}$ is a measure of the degree of homopolar binding. According to Burstein and Smith $\lambda_{0}$ should attain a maximum value for intermediate bonding, i.e., bonding containing appreciable amounts of both ionic and homopolar bonding, and decrease as the bonding becomes either more ionic or more homopolar. The present studies do not agree with this conclusion, the value of $\lambda_{0}$ being largest for the most covalent crystal-diamond. It appears that the conclusions of Burstein and Smith were based upon erroneous data for diamond.

For the glasses, calculated values of $\lambda_{0}$ are also given in table 2 . These values show little variation for the three glasses but it is noteworthy that $\lambda_{0}$ data calculated by both the Lorentz-Lorenz and the Drude equations are positive. Previous data on liquids show that $\lambda_{0}$ is positive when computed from the L-L equation but negative when computed from the Drude equation [30]. This behavior in liquids was explained as arising from the change in the local field brought about by compression of the liquids

3 Krishan and Roy [40] have shown the equivalence of the Lorentz-Lorenz and Drude equations in expressing the frequency dependence of the refractive index and have shown that the Drude expression accounts for the local field by incorporating its effect into the value of the fundamental absorption frequency. However, eq (9) is a simplified version of the generalized Drude equation and is valid only at a single frequency. When written in the form of eq (9) it is implied that the change in the local field with density has been ignored. 
and incorporated into a change in the fundamental absorption frequency in the Drude model. This greater change in the local field of the liquids arises from their greater compressibilities.

Ramachandran and Radhakrishnan $[41,6]$ have shown that the thermal properties of the index of refraction may be understood by considering the change of index with temperature to consist of three independent contributions which they label $P, Q$, and $R$. $P$ represents the change arising only from a change in the number of scattering centers and is a pure density effect, $Q$ represents the change in polarizability resulting from the density change, and $R$ consists of the effect of temperature alone on the polarizability. Recognizing that $d \mu / d T=P+Q+R$, the values they derived using the Drude equation are as follows:

$$
\begin{aligned}
& P=-\gamma\left(\frac{\mu^{2}-1}{2 \mu}\right) \\
& Q=-\gamma\left(\rho \frac{d \mu}{d \rho}-\frac{\mu^{2}-1}{2 \mu}\right) \\
& R=\frac{d \mu}{d T}+\gamma \rho \frac{d \mu}{d \rho}
\end{aligned}
$$

where $\gamma=-\frac{1}{\rho}\left(\frac{d \rho}{d T}\right)_{P}$. Values of $\rho d \mu / d \rho$ they used $[41,6]$ were evaluated from the elasto-optic constants (eq (3)). The quantities $P, Q$, and $R$ have been re- evaluated using the same data that Krishnan and Roy used except that experimental values of $\rho \Delta \mu / \Delta \rho$ have been substituted for $\rho d \mu / d \rho$. [There is one further change; it appears that the earlier results were calculated using an erroneous value for the volume expansivity of diamond, $1.3 \times 10^{-5} /{ }^{\circ} \mathrm{C}[41,6]$ compared to the correct value, $0.31 \times 10^{-5} /{ }^{\circ} \mathrm{C}[42,9]$.] The results are given in table 4 . The present data agree reasonably well with the earlier results in most instances, there being slight changes in $Q$ and $R$ resulting from the present values of $\Delta \mu / \Delta \rho$. There is a large change, however, in the results for diamond.

It is also possible to calculate $R$, which is defined as $(d \mu / d T)_{V}$, from constant volume data. For a given material the calculations involve finding a temperature at $1 \mathrm{~atm}$ where the specific volume is the same as that at $25{ }^{\circ} \mathrm{C}$ and an elevated pressure; the refractive indices which pertain for the two different situations may then be used to find $R$. Such calculations have been made using values of $\gamma$ and $(d \mu / d T)_{P}$ as a function of temperature. The original sources for the values of $\gamma$ and $(d \mu / d T)_{P}$ for the different solids have been identified in the list of references. ${ }^{4}$ Much of the necessary information may be found in the compilation made by Krishnan [6]. The values are given in the column headed $R$ (experimental) in table 4 . It can be seen that there is reasonably good agreement between corresponding values of $R$ in the two columns.

${ }^{4} \mathrm{BSC} 517 / 645$ is known to have the same nominal composition as the glass

\begin{tabular}{|c|c|c|c|c|c|c|c|c|c|c|c|c|}
\hline Material & ${ }^{\mu}{ }_{D}$ & $\gamma \times 10^{5}$ & $d \mu / d T \times 10^{5}$ & $P \times 10^{5}$ & $Q \times 10^{5}$ & $R \times 10^{5}$ & $\begin{array}{c}R \times 10^{5} \\
\text { (experi- } \\
\text { mental) }\end{array}$ & $\left(\frac{V}{\alpha} \frac{\partial \alpha}{\partial V}\right)_{T}$ & $\left(\frac{V}{\alpha} \frac{\partial \alpha}{\partial V}-1\right)_{T}$ & $\left(\frac{V}{\alpha} \frac{\partial \alpha}{\partial V}-1\right)_{T} \gamma$ & $\underset{\quad\left(\frac{1}{\alpha} \frac{\partial \alpha}{\partial T}\right)_{V}}{\times 10^{5}}$ & $\begin{array}{c}\left(\frac{1}{\alpha} \frac{\partial \alpha}{\partial T}\right)_{V} \\
\times 10^{5} \\
(\text { experi- } \\
\text { mental) }\end{array}$ \\
\hline $\begin{array}{l}\mathrm{KBr} \\
\mathrm{NaCl} \\
\mathrm{LiF} \\
\mathrm{MgO} \\
\text { Diamond } \\
\text { Fused SiO } \\
\text { BSC 517/645... } \\
\text { Plate glass }\end{array}$ & $\begin{array}{l}1.559 \\
1.544 \\
1.392 \\
1.736 \\
2.417 \\
1.458 \\
1.517 \\
1.518\end{array}$ & $\begin{array}{c}{ }^{\circ} C^{-1} \\
12.0 \\
12.0 \\
10.2 \\
3.9 \\
0.31 \\
.15 \\
2.0 \\
1.9\end{array}$ & $\begin{array}{c}{ }^{\circ} C^{-1} \\
-3.6 \\
-3.8 \\
-1.3 \\
1.6 \\
1.0 \\
0.92 \\
.17 \\
.27\end{array}$ & $\begin{array}{l}{ }^{\circ} C^{-1} \\
-5.5 \\
-5.4 \\
-3.4 \\
-2.3 \\
-0.3 \\
-.06 \\
-.9 \\
-.8\end{array}$ & $\begin{array}{c}{ }^{\circ} C^{-1} \\
1.3 \\
2.0 \\
3.4 \\
3.5 \\
0.8 \\
.01 \\
.2 \\
.2\end{array}$ & $\begin{array}{c}{ }^{\circ} C^{-1} \\
0.6 \\
-0.4 \\
-1.3 \\
0.4 \\
.5 \\
.97 \\
.8 \\
.9\end{array}$ & $\begin{array}{r}{ }^{\circ} \mathrm{C}^{-1} \\
0.4 \\
-.3 \\
-1.3 \\
0.6 \\
.6 \\
1.0 \\
.7\end{array}$ & $\begin{array}{l}0.2 \\
.4 \\
1.0 \\
1.5 \\
1.7 \\
0.2 \\
.2 \\
.3\end{array}$ & $\begin{array}{r}-0.8 \\
-.7 \\
0.0 \\
.5 \\
.7 \\
-.8 \\
-.8 \\
-.7\end{array}$ & $\begin{array}{r}{ }^{\circ} C^{-1} \\
-9.4 \\
-7.8 \\
0.0 \\
2.0 \\
0.2 \\
-.1 \\
-1.5 \\
-1.4\end{array}$ & $\begin{array}{r}{ }^{\circ} \mathrm{C}^{-1} \\
1.6 \\
-.7 \\
-3.8 \\
0.7 \\
.8 \\
2.5 \\
1.9 \\
2.0\end{array}$ & $\begin{array}{r}{ }^{\circ} \mathrm{C}^{-1} \\
0.9 \\
-.7 \\
-3.9 \\
1.0 \\
0.6 \\
2.6 \\
1.5\end{array}$ \\
\hline
\end{tabular}
designated as $\mathrm{BSC}-2$ in reference [8].

TABLE 4. Analysis of change in refractive index and polarizability for cubic crystals and glasses

For the present purposes where volume and temperature may both be considered as independent variables it appears more desirable to change the analysis slightly as follows: Starting with the Drude relationship (eq (9)) and considering the polarizability $\alpha_{2}$ to be a function of $V$ and $T$, it can be shown that

$$
d \mu / \mu=\frac{\left(\mu^{2}-1\right)}{2 \mu^{2}}\left[\frac{1}{\alpha}\left(\frac{\partial \alpha}{\partial T}\right)_{V} d T+\left(\frac{V}{\alpha}\left(\frac{\partial \alpha}{\partial V}\right)_{T}-1\right) \frac{d V}{V}\right]
$$

(In eq (16) and the treatment that follows $\alpha_{2}$ is written simply as $\alpha$.) In this expression $\frac{1}{\alpha}\left(\frac{\partial \alpha}{\partial T}\right)_{V}$ is the pure temperature coefficient of polarizability, $\frac{V}{\alpha}\left(\frac{\partial \alpha}{\partial V}\right)_{T}$ is the pure volume coefficient of polarizability and the term $\left(\frac{V}{\alpha}\left(\frac{\partial \alpha}{\partial V}\right)_{T}-1\right)$ represents the total contribution of volume to the change in index. At present the term $\frac{V}{\alpha}\left(\frac{\partial \alpha}{\partial V}\right)_{T}$ can be evaluated from the constant temperature experiments. To obtain $\frac{1}{\alpha}\left(\frac{\partial \alpha}{\partial T}\right)_{V}$ the volume is taken to be a function of $P$ and $T$. Then $d V=\left(\frac{\partial V}{\partial T}\right)_{p} d T+\left(\frac{\partial V}{\partial P}\right)_{T} d P$ and using 
$\frac{1}{V}\left(\frac{d V}{d T}\right)_{P}=\gamma$ and $-\frac{1}{V}\left(\frac{d V}{d P}\right)_{T}=\beta$ eq (16) can be recast in the form

$$
\begin{array}{r}
\frac{d \mu}{\mu}=\frac{\mu^{2}-1}{2 \mu^{2}}\left[\frac{1}{\alpha}\left(\frac{\partial \alpha}{\partial T}\right)_{V} d T+(\gamma d T-\beta d P)\right. \\
\left.\left(\frac{V}{\alpha}\left(\frac{\partial \alpha}{\partial V}\right)_{T}-1\right)\right] .
\end{array}
$$

The value of $V / \alpha(\partial \alpha / \partial V)_{T}$ determined from eq (16) can be combined with available data on $\gamma$ and on the temperature variation of index at constant pressure to derive values for $\frac{1}{\alpha}\left(\frac{\partial \alpha}{\partial T}\right)_{V}$. If the LorentzLorenz equation is used the same expression will be obtained except that the factor $\frac{\left(\mu^{2}-1\right)\left(\mu^{2}+2\right)}{6 \mu^{2}}$ replaces $\frac{\mu^{2}-1}{2 \mu^{2}}$. This represents only a different scaling factor and in no way affects the relative magnitudes of the terms in the brackets which are of primary interest.

The calculated values of the terms of interest in eq (17) are given in table 4 . The volume coefficient of polarizability is seen to be positive in all materials as expected. The positive sign means a decreased polarizability on compression and an increased polarizability on heating arising from the thermal expansion. In the alkali halides the numerical values of the coefficient increase as the anion packing increases. Values for $\mathrm{MgO}$ and diamond are the largest in the table, a result consistent with the large interatomic interactions presumed to occur in these materials. The small values for the glasses are to be expected from the smaller interatomic interactions expected for such open structures. The smallest value in the table applies to fused $\mathrm{SiO}_{2}$ which has the most "open" structure. (The figures in table 4 have been rounded off and this conclusion is not obvious from the data given.) The total volume contribution is given in the next column and these values are generally negative except for diamond and $\mathrm{MgO}$. It is noteworthy that in every case the volume coefficient of polarizability represents an appreciable portion of the total volume effect. It must be concluded that any relationship between index and density which ignores the effect of this contribution to the index can, at best, be only approximately correct. The next column tabulates the total volume term multiplied by the expansivity to permit comparison on an equal basis with values for the temperature coefficients of polarizability given in the next to last column. The temperature coefficients vary in both sign and size with the value apparently decreasing with packing in the alkali halides and being rather large positively in the glasses. There are insufficient data, however, to verify that these conclusions are typical. $\left(\frac{1}{\alpha} \frac{\partial \alpha}{\partial T}\right)_{v}$ may also be found by substituting values of $(d \mu / d T)_{v}$ in eq (16). These values are shown in the last column of table 4. Again it may be seen that there is fairly good agreement between corresponding values in the two columns of figures.

With the data given in table 4 it is instructive to consider the change in index resulting from two separate experiments, first an isothermal compression and second an isobaric temperature change. An isothermal compression produces an increase in index in $\mathrm{KBr}, \mathrm{NaCl}$, and all the glasses because the predominant effect is an increase in the number of scattering centers per unit volume. The decrease in polarizability with decreasing volume is less than the density effect in these materials. In LiF the index undergoes no change (to $1 \mathrm{kbar}$ ) because the density increase and the polarizability decrease exactly balance. In both $\mathrm{MgO}$ and diamond the index decreases on compression, the polarizability decrease overbalancing the density increase.

It is of interest to consider the effect of pressures higher than the $1 \mathrm{kbar}$ used here. It appears likely that the volume coefficient of polarizability will increase as the volume decreases because the interatomic interactions should increase in magnitude. In the absence of other effects, this requires that at some elevated pressure the indices of the solids which increased with pressure at low pressures will pass through a maximum value and then decrease at still higher pressures. Further the decrease in index should accelerate at still higher pressures. The pressures required to produce these changes are not obvious but plans are being made to extend these measurements to $10 \mathrm{~kb}$. In this range it appears very probable that a decrease might be expected for LiF. For the other alkali halides a reversal or a decrease in the rate of change of index with density might be expected.

An isobaric temperature increase results in a decrease in indices for $\mathrm{KBr}, \mathrm{NaCl}$, and $\mathrm{LiF}$ but an increase in indices for $\mathrm{MgO}$, diamond and all the glasses. Although the resultant effects are similar for members in each of the two groups, there are various causes, and it is necessary to consider the details of the process. In $\mathrm{KBr}$ the volume expansion causes a decrease in index because the number of scattering centers per unit volume decreases. This effect is great enough to overbalance the increased polarizabilities arising from the increased volume and temperature. In $\mathrm{NaCl}$ the temperature coefficient acts in conjunction with the density effect to decrease the index. In $\mathrm{LiF}$ there is no total volume contribution and the decrease in index arises solely from the temperature coefficient of polarizability. In both $\mathrm{MgO}$ and diamond the index increases because the positive temperature and volume coefficients of polarizability overbalance the negative contribution from the decrease in the number of scatterers with the volume coefficient playing the major role. All the glasses show an increase in index because the comparatively large temperature coefficient of polarizability outweighs the negative effect of the total contribution of change in volume which is nearly as large. 
The glasses require further consideration because it is known [8] that the index-temperature relationships of most optical glasses exhibit a minimum in index. The glass BSC-517/645 has such a minimum near $-80{ }^{\circ} \mathrm{C}$ and although data on plate glass do not appear to be available a minimum may reasonably be expected at some temperature below $25{ }^{\circ} \mathrm{C}$. On the other hand the index of refraction of fused $\mathrm{SiO}_{2}$ is known to decrease monotonically with decreasing temperature $[51,16]$ despite the reversal of its coefficient of thermal expansion. A reasonably sound explanation of these effects requires data on the two terms $\frac{v}{\alpha} \frac{\partial^{2} \alpha}{\partial V \partial T}$ and $\frac{1}{\alpha} \frac{\partial^{2} \alpha}{\partial T^{2}}$ which are apparently not available at this time but certain conclusions may be of interest until such data are obtained. It might be expected that for normal materials the polarizability arising from pure thermal agitation should decrease at higher temperatures because of increased interaction. However, it is not apparent whether

$\frac{\partial \alpha}{\partial T}$ increases or decreases. Data by Radhakrishnan on LiF [49] show that $d \mu / d T$ becomes increasingly negative at higher temperatures. At room temperature this crystal shows a zero volume contribution, and, if it is assumed that this contribution is negligibly small at elevated temperatures, it may be concluded that $\frac{1}{\alpha}\left(\frac{\partial^{2} \alpha}{\partial T^{2}}\right)$ is negative. The glasses, however, are not normal solids so that it is not certain that they exhibit a similar behavior. It is known that $\frac{1}{V_{0}}\left(\frac{\partial^{2} V}{\partial T^{2}}\right)_{p}$ is positive for the glasses and it is to be expected that $\frac{V}{\alpha}\left(\frac{\partial \alpha}{\partial V}\right)_{T}$ would increase somewhat at low temperatures because of the closer packing.

In table 4 it is observed that for BSC $517 / 645$ and plate glass that the total volume contributions and the temperature coefficients of polarizability are of opposite signs but nearly equal in absolute values. If either one or both of those quantities changes slightly in the proper direction on lowering the temperature it is clear that the sign of $d \mu / d T$ will reverse. Since this reversal occurs below room temperature in BSC $517 / 645$ and is expected in a similar temperature range for plate glass, it is probable that the temperature coefficient of polarizability decreases with decreasing temperature in these glasses in contrast with the opposite behavior observed in LiF. The situation in fused $\mathrm{SiO}_{2}$ is somewhat different in that $\frac{1}{\alpha}\left(\frac{\partial \alpha}{\partial T}\right)$ is at least ten times as great as the total volume effect although they are of opposite sign. In this situation a small change in either quantity will be insufficient to reverse the sign of $d \mu / d T$. In addition when the expansivity of fused $\mathrm{SiO}_{2}$ becomes negative both the volume term and $\frac{1}{\alpha} \frac{\partial \alpha}{\partial T}$ are positive and cannot cancel. It may be noted that the interpretation here is similar to that advanced by Prod'homme [52] for the anomalous temperature-index behavior in optical glasses but is somewhat more detailed. It appears that the positive value of $d \mu / d T$ in optical glasses arises from the comparatively large temperature coefficient of polarizability coupled with the low coefficient of thermal expansion. Since these quantities are not common to all glasses [52] it appears that they must be attributed to the silica network.

The authors are indebted to Harry B. Williams of the National Bureau of Standards for his great help in the preparation of specimens. The authors also thank Edward N. Farabaugh and William S. Brower of the National Bureau of Standards for their help in the orientation of the uniaxial crystals.

\section{References}

[1] E. Burstein and P. L. Smith, Proc. Ind. Acad. Sci A28, 377 (1948)

[2] H. Mueller, Phys. Rev. 47, 947 (1935).

[3] H. Mueller, Physies 47, No. 6, 179 (1935).

[4] H. N. Ritland, J. Am. Ceram. Soc. 38, 86 (1955).

[5] C. Weir, S. Spinner, I. Malitson, and W. Rodney, J. Res. NBS $\mathbf{5 8}$ No. 4, 189 (1957) RP2751.

[6] R. S. Krishnan, Progress in Crystal Physics, Volume I (Interscience Publishers, New York, London, 1958).

17] M. A. Jeppensen, J. Opt. Soc. Am. 48 No. 9, 629 (1958).

[8] (Thermal expansion and $d u / d T$ data on BSC 517/645)F. A. Molby, J. Opt. Soc. Am. 39, No. 7, 600 (1949).

[9] ( $d \mathrm{u} / d T$ data on diamond)-G. N. Ramachandran, Proc. Ind. Acad. Sci. A25, 266 (1947).

[10] R. M. Waxler and C. E. Weir, J. Res. NBS 6yA (Phys. and Chem.) No. 2, 163 (1963).

[11] (Refractive index and $d u / d T$ data on $\mathrm{KBr}$ )-R. J. Spindler and W. S. Rodney, J. Res. NBS 49 No. 4, 253 (1952) RP2301.

[12] (Refractive index data on $\mathrm{NaCl}$ ) - F. Kohlrausch, Praktische Physik II B. G. Teubner, Leipzig, 528 (1943).

[13] (Refractive index data on LiF) - L. W. Tilton and E. K. Plyler, J. Res. NBS 47 No. 1, 25 (1951) RP2223.

[14] (Refractive index data on diamond)-F. Peter, Z. Phys. 15, 358 (1923).

[15] (Refractive index data on $\mathrm{MgO}$ ) - R. E. Stephens and I. H. Malitson, J. Res. NBS 49 No. 4, 249 (1952) RP2360.

[16] (Refractive index data on quartz, and thermal expansion and $d \mathrm{u} / d T$ data on fused silica)-R. B. Sosman, The Properties of Silica, (Chem. Cat. Co. N.Y., 1927).

[17] (Refractive index data on $\mathrm{Al}_{2} \mathrm{O}_{3}$ ) - R. W. Kebler, Optical Properties of Synthetic Sapphire (Iinde Co., N.Y.).

[18] (Refractive index data on fused silica)-J. B. Austin, Physics 3240 (1932).

[19] (Refractive index data on BSC 517/645) - R. E. Stephens and W. S. Rodney, J. Res. NBS, 52 No. 6, 303 (1954) RP2504.

[20] (Refractive index data on plate glass) - E. Hamilton, R. Waxler and J. M. Nivert, Jr., Optical Glass Technology Development, NBS Report No. 5440 (1957).

[21] (Compression data on $\mathrm{KBr}$, NaCl and $\mathrm{LiF}$ ) - P. W. Bridgman, The Physics of High Pressure (G. Bell \& Sons, Ltd., London 1949).

[22] (Compression data on quartz) - P. W. Bridgman, Am. J. Sci. Fifth series 15 No. 88, 287 (1928).

[23] (Compression data on $\mathrm{Al}_{2} \mathrm{O}_{3}$ ) - P. W. Bridgman, Proc. Am. Acad. Arts Sci. 68, 27 (1932).

[24] (Compression data on fused silica)-P. W. Bridgman, Am. J. Sci. 10, 359 (1925).

[25] (Compression data on diamond and plate glass)-L. H. Adams and E. D. Williamson, J. Frank Inst. 195, 475 (1923). 
[26] (Compression data on MgO) - C. E. Weir, J. Res. NBS 56 No. 4, 187 (1956) RP2666.

[27] S. Spinner, J. Am. Cer. Soc. 37 [5], 229 (1954).

[28] F. A. Jenkins and H. E. White, Fundamentals of Optics (McGraw-Hill Book Company, Inc., New York, Toronto, London, 1950).

[29] E. S. Jog, Jour. Ind. Inst. Sci. 39A, 101 (1957).

[30] R. M. Waxler, C. E. Weir, and H. W. Schamp, Jr., J. Res. NBS 68A (Phys. and Chem.) No. 5, 489 (1964).

[31] F. Pockels, Ann. Phys. 37, 151 (1889).

[32] D. F. Gibbs and G. J. Hill, Phil. Mag. 8, 367 (1963).

[33] C. Schaefer and H. Nassenstein, Z. Naturforsch. [A] 8, 90 (1953).

[34] K. Vedam, Proc. Ind. Acad. Sci. [A] 31, 450 (1950).

[35] F. Pockels, Ann. Phys. Chem. 37 (4), 269 (1889).

[36] R. M. Waxler and A. Napolitano, J. Res. NBS 59 No. 2, 121 (1957) RP2779

[37] R. M. Waxler, Glass Ind. 34, 258 (1953)

[38] S. Spinner, personal communication.

[39] F. Pockels, Ann. d. Phys. 7, 745 (1902).

[40] K. S. Krishnan and S. K. Roy, Phil. Mag. 43, 1000 (1952).

[41] G. N. Ramachandran and T. Radhakrishnan, Phil. Mag. 43, 317 (1952)

[42] (Thermal expansion data on diamond)-R. S. Krishnan, Proc. Indian Acad. Sci. A24, 33 (1946).
[43] (Thermal expansion data on KBr) - R. Srinivasan, J. Indian Inst. Sci. A37, 232 (1955).

[44] (Thermal expansion data on $\mathrm{NaCl}$ ) - R. M. Buffington and W. M. Latimer, J. Amer. Chem. Soc. 48, 2305 (1926).

[45] (Thermal expansion data on LiF)-A. Eucken and W. Dannöhl, Z. Electrochem. 40, 814 (1934).

[46] (Thermal expansion data on $\mathrm{MgO}$ )-M. Durand, Physics , 297 (1936).

[47] (Thermal expansion data on fused silica) - K. Scheel and W. Heuse, Verh. Deutsch. Physik. Ges. 161 (1914).

[48] (du/dT data on $\mathrm{NaCl})$ - F. J. Micheli, Arch. Sc. Phys. Nat. 13, 217 (1902).

[49] (du/dT data on $\mathrm{LiF})$ - T. Radhakrishnan, Proc. Indian Acad. Sci. A31, 224 (1950).

[50] (du/dT data on MgO) - T. Radhakrishnan, Proc. Indian Acad. Sci. A33, 22 (1951).

[51] ( $d u / d T$ data on fused silica)-F. Rinne, Neues Jahrb. Mineral. Beil. Bd. 39, 388 (1914).

[52] L. Prod'homme, Phys. and Chem. of Glasses 1 No. 4, $119(1960)$

(Paper 69A4-352) 